\title{
Automated Water Tank Overflow Control Unit Integrated with Mobile Application
}

\author{
S.Gowri, Pola Pranathi, Kodali Sravya \\ Faculty of Computing, Dept. of IT., Sathyabama University, Chennai- 600119, India.
}

\begin{abstract}
-
Basically automation in systems would help reduce wastage of resources in various ways, one such way for the prevention of wastage of water resource to control the water tanks from overflow. In this automated world, the handling of any kind of devices with comfort is made to be the easiest factor everywhere. Considering this aspect of comfort zones and the prevention of wastage of resources, a design of an automated overflow control circuit unit was proposed in this paper. The proposal was designed on the perspective of controlling the flow of water into the tanks automatically, by setting the requirements as per the user needs using a Mobile Application in android. The connectivity of the circuit and the application is accomplished using Bluetooth device for handling the communication between the mobile and the overflow circuit. The entire system was tested in its operation and was scrutinized on the whole.
\end{abstract}

\section{I.INTRODUCTION}

As days passed by the growth of technology would seem to be a major aspect bound to humans and machines. The growth of this technology reflects in all the aspects of the day to day life of a human being. Technology plays a vital role in every moment in their entire life span of a human, from a just born baby to a very old man. Technology has almost scatted over all the sectors, from small scale home usage to a large scale satellite. If a closer view was taken upon the technology, every step we make or every move we do deals with the technology.

We take the system of water tank for imparting the new technology using sensors and we overcome the flaws of present systems in the water tank control unit.

According to the present statics the wastage of water due to overflow of tanks has a major impact on the water scarcity, in the present scenario of a busy world the manual monitoring of water filling in the tanks seems to have a difficulty in switching ON and OFF of the motor, due to which the wastage of water occurs, there are a plenty of ways to prevent using the current technology yet they do not show a much impact over the prevention.

A few disadvantages of the present mechanism of the overflow control systems are:

- Ball Mechanism: loosing of its mechanism after a period of time.
- Fuzzy: requirement of too many complications in setting up the system.

- Radio Frequency Communication based: requirement of manual view.

To overcome such disadvantages a design of a system which is automated as well as manually controlled (according to the user requirement) is designed.

\section{II.RELATED WORKS}

Sanam Pudasaini et al. (2014) presented system of a programmed water level controller with SMS notice. Their task was done to help client in burden shedding based nation like Nepal. SMS notice was added to program controller framework with the goal that water can be overseen by client amid burden shedding. Two frameworks work synergistically; programmed level controller framework and SMS framework. The system was produced in Arduino project creating environment and transferred to the Microcontroller. Water level in the framework is controlled naturally. The controller works on a battery power. At whatever point the framework experiences void level and the status of burden shedding, the SMS warning is sent to the client.

S. B. Jagadal and S. V. Halse (2013) proposed a control framework which can be utilized to screen numerous tanks by controlling the operation of engines which pumps water from sump to the overhead tanks utilizing a solitary $8051 \mathrm{MCU}$ interfaced with LCD show in a virtual situation. Here they presented a multi-tank 
control framework with four tanks to be observed. A low level computing construct project was created to control these four engines in both modes programmed and also manual. They have utilized rationale control outline to choose the specific tank to fill the water to yearning level. There is likewise an abnormal state alert which is utilized to keep the flood of the water. There is a programmed fill alternative which fills every one of the tanks one by one in a consecutive way naturally. This control framework additionally permits client to fill the longing tank utilizing select and run catch of the separate engine in an arbitrary succession. At the point when the framework is dynamic and running, it can show the water level through visual diagram LED when associated with sensor point. A LCD showcase of size 40X2 is interfaced to 8051 MCS to show the status of the tank other than giving important guideline to work this framework. They send the project to LCD from $8051 \mathrm{MCS}$. This LCD is 40 characters wide with two lines. This control framework schematic is in view of essential computerized idea to arrange the circuit outline.

Muktha Shankari K et al. (2013) discussed about configuration and usage of water level control framework which is remote, programmed, financially savvy and solid. It utilizes two Radio Frequency handsets alongside a controller each introduced at the tank and sump. Radio Frequency handsets are utilized for remote correspondence. It is totally mechanized with the assistance of a small scale controller. The framework needn't bother with any consideration of the client unless the sump is unfilled. Establishment expense is decreased following the framework is remote. It is solid in light of the fact that it has no issues emerging after establishment, for example, breakage of wire.

Jiang Wei (2010) presented a fuzzy control hypothesis which is utilized to build up a liquid level, and the liquid info stream rate to focus the measure of control, which to push the valve's opening size and pace so as to control the water supply valve opening change level, so that the fluid position of compartment keeps up a consistent, and the MATLAB programming is utilized to confirm the impact of fuzzy control framework. His outcomes demonstrate that in this fuzzy control framework the advantages of monetary operation of water supply can be guaranteed by guaranteeing a consistent level of fluid.

\section{OVERFLOW CONTROL SYSTEM DESCRIPTION}

The System comprises of the following components:

1. 8051 microcontroller which acts as a brain to the circuit working according to the embedded instructions

2. A blue tooth device which plays the role of connectivity in communication between the system and the mobile application (send notification and receiving any manual instruction sent through the application when set to manual operating mode)

3. A three level sensing terminal which is fixed inside the tank to have a control on the level of the water flowing into the tank, the three levels are:
a) FULL
b) MIDIUM
c) LOW

4. Application for mobiles which is installed in the mobile phone (This is optional, the system works both on manual and automatic, people with no smart phones would have to choose the automatic only and for the manual monitoring a display board with a controller could be replaced in the place of the mobile application

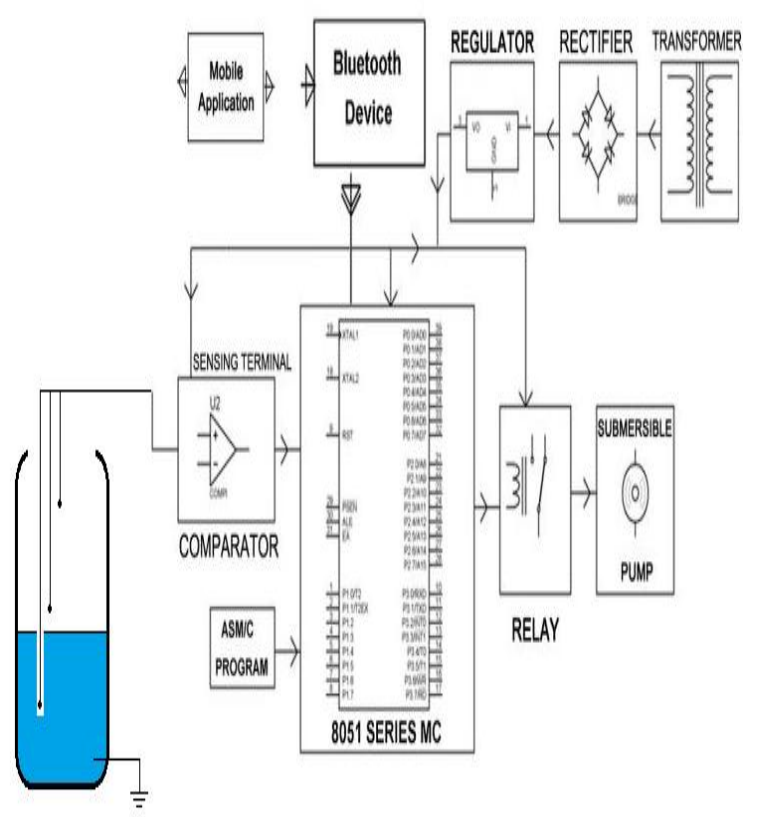

Fig 1. Overflow Control System Circuit

This control system plays a vital role in conservation of water, the automated water filling mode is present were the moisture of the water in the tank is checked 
using a three level sensor which indicates the level of water filled and water motor is switched $\mathrm{ON}$ and switched OFF according to the moisture level presence according to the set level of filling requirement. This unit also has a manual switch OFF and ON mechanism for the users in order to avoid water flow when people go out of station or so.

\section{CONCLUSION}

This work brings in technology into the house by mechanizing of household requirements, which make the work of water storage system much easier and convenient over handling. The overall system has a great impact in the prevention of water wastage more effective than the manual work.

The most important merits of the system are:

(i) The conservation of water resource

(ii) Manual effort reduction

(iii) Time to time alters over the status water storage.

\section{REFERENCES}

[1] Md. Moyeed Abrar1, Rajendra.R.Patil2, "Logic Gate Based Automatic Water Level Controller", IJRET: International Journal of Research in Engineering and Technology, Volume 03, Issue 04, pp. 477-482, 2014.

[2] Sanam Pudasaini, Anuj Pathak, Sukirti Dhakal and Milan Paudel, "Automatic Water Level Controller with Short Messaging Service (SMS) Notification", International Journal of Scientific and Research Publications, Volume 4, Issue 9, pp. 1-4, 2014.
[3] Muktha Shankari, Jyothi, Manu E, Naveen I and Harsha Herle, "Wireless Automatic Water Level Control using Radio Frequency Communication", International Journal of Advanced Research in Electrical, Electronics and Instrumentation Engineering, Vol. 2, Issue 4, pp. 1320-1324, 2013.

[4] S. B. Jagadal and s. V. Halse, "8051 Microcontroller Based Multiple Water Tank Control System" Journal of Computer and Mathematical Sciences Vol. 4, Issue 5, pp. 382-401, 2013.

[5] S. M. Khaled Reza, Shah Ahsanuzzaman Md. Tariq and S.M. Mohsin Reza "Microcontroller Based Automated Water Level Sensing and Controlling: Design and Implementation Issue" Proceedings of the World Congress on Engineering and Computer Science, Vol. I, 2010.

[6] Pan Lingfeng, $\mathrm{Hu} \mathrm{Ji}$ and Zhiwang $\mathrm{Xu}$, "Design and realization of a intelligent mixed water valve control system", International Conference on Electrical and Control Engineering (ICECE), pp. 5816-5818, 2011.

[7] Tingjun Wang and Li Gu, "An Electronic Water-Saving Integrated System for Toilet Bowl Based on Electromagnetic Valve Controlling", Second International Conference on Information and Computing Science ICIC '09, pp. 258 - 261, 2009.

[8] Jiang Wei, "Intelligent Building Control of Water Tank Based on Fuzzy Theory", International Conference on Intelligent Computation Technology and Automation (ICICTA), pp. 549 $-552,2010$ 